\title{
La présence de la Folie dans les Oeuvres de Louise Labé
}

\author{
WILSON BALDRIDGE
}

Cette brève étude propose une lecture des images de la folie qui traversent de part en part l'écriture passionnée de Louise Labé. Ces images traduisent l'essence même de l'amour, cette sortie hors-de-soi, dans l'échange affectif total avec l'autre, qui "étrange" (écarte, éloigne) les amants d'eux-mêmes en leur faisant connaître une force libératrice supérieure à toute raison. ${ }^{1}$ Cette puissance de la folie amoureuse, qui unit les amants corps et âme l'un à l'autre, figure implicitement l'unité formelle du monde de la ressemblance poétique.

En faisant allusion à "la belle première naissance d'Amour" comme indice à la fois historique, mythique et psychologique, il s'agit ici de retracer la présence de la folie dans les Oeuvres de Labé, c'est-à-dire de suivre à la trace le rapport entre Folie comme personnage féminin, signe entre autres de la passion effrénée, et l'imaginaire comme milieu de la figuration. ${ }^{2}$ Il faut entendre ici le nom de "Folie" non pas dans son acception médicale en tant que maladie ou lésion des facultés intellectuelles et affectives, mais au contraire comme une affirmation quasi sacrée de l'émotivité et de l'imaginaire créateur. L'oeuvre poétique de Labé exprime une sagesse de la folie. Une telle valorisation des paradoxes de la passion, de la fantaisie et du délire prend le contre-pied de l'amour de la sagesse qui, depuis l'antiquité, aura défini la philosophie idéaliste masculine.

La question critique qui sous-tend cette étude de la folie amoureuse vise l'articulation du motif de la différence dans une écriture au fémininin. ${ }^{3} L$ 'Epître dédicatoire placée par Labé en tête de ses Oeuvres expose l'essentiel de la pensée féministe de l'auteure aussi bien que sa conception de la différence entre l'activité d'écrire et les autres "récréations" (282). S'adressant à toutes ses lectrices à travers la dédicataire Clémence de Bourges, Labé précise la valeur (fondée sur la perception du même à l'intérieur de la différence temporelle) de la pratique de l'écriture: 
Mais quand il advient que mettons par écrit nos conceptions, combien que puis après notre cerveau courre par une infinité d'affaires et incessamment remue, si est-ce que, longtemps après reprenant nos écrits, nous revenons au même point, et à la même disposition où nous étions. Lors nous redouble notre aise, car nous retrouvons le plaisir passé qu'avons eu ou en la matière dont écrivions, ou en l'intelligence des sciences où lors étions adonnés. Et outre ce, le jugement que font nos secondes conceptions des premières, nous rend un singulier contentement. ${ }^{4}$

Nous examinerons le motif de la folie d'abord dans le Débat de Folie et d'Amour, puis dans les sonnets VIII, XVII et XVIII où passion et déraison s'entrelacent à travers les vocables de la persona lyrique. L'Argument du Débat constitue un point de départ efficace pour l'analyse du rapport folie/amour comme trope pour la complexion du langage poétique:

Jupiter faisait un grand festin, où était commandé à tous les Dieux se trouver. Amour et Folie arrivent au même instant sur la porte du Palais: laquelle étant jà fermée, et n'ayant que le guichet ouvert, Folie voyant Amour jà prêt à mettre un pied dedans, s'avance et passe la première. Amour se voyant poussé, entre en colère: Folie soutient lui appartenir de passer devant. Ils entrent en dispute sur leurs puissances, dignités et préséances. Amour ne la pouvant vaincre de paroles, met la main à son arc, et lui lâche une flèche, mais en vain: pource que Folie soudain se rend invisible: et se voulant venger, ôte les yeux à Amour. Et pour couvrir le lieu où ils étaient, lui mit un bandeau fait de tel artifice, qu'impossible est lui ôter. Vénus se plaint de Folie, Jupiter veut entendre leur différend. Apollon et Mercure débattent le droit de l'une et l'autre partie. Jupiter les ayant longuement ouis, en demande l'opinion aux Dieux: puis prononce sa sentence. (287)

Plaidant en faveur d'Amour, Apollon s'efforce de montrer que la folie ne peut être que la ruine de tout amour, celui-ci entendu non seulement comme eros mais comme principe universel de la paix et de l'harmonie. De plus, le dieu solaire affirme que Folie constitue une menace à l'autorité de Jupiter et donc à la hiérarchie divine, et demande par conséquent que la fille de Jeunesse soit bannic des environs immédiats d'Amour. Pour parer à la menace d'un rapprochement entre Folic et Amour, Apollon pric les dieux assemblés de restituer les yeux au fils de Vénus, car si Amour s'en prend au cocur, Folic s'attaque à la vue et à l'esprit, provoquant un aveuglement qui, généralisé, finirait par entraîner un effacement catastrophique de toutes les distinctions au monde.

Lorsque Mercure prend la parole pour défendre Folie, il se donne trois buts: "Défendre la tête de Folie, contre laquelle Amour a juré: répondre 
aux accusations que j'entends être faites à Folie: et à la demande qu'il fait de ses yeux" (326-27). Mercure aura reconnu la demande d'exclusion de Folie comme le postulat essentiel de l'argument d'Apollon, et le messager des dieux organise son plaidoyer autour du thème de l'unité originaire de Folie et d'Amour, répétant de diverses manières que le fils de Vénus ne pourrait absolument pas exister sans Folie, que celle-ci incarne en fait la source intime du pouvoir d'Amour. Mercure affirme que Folie et Amour sont amis, de tous temps "unis et conjoints" (326): répondant aux demandes d'Apollon, Mercure donne à entendre qu'une certaine perte de la raison constitue la condition de possibilité de tout amour. Mercure dit: "Mon intention sera de montrer qu'en tout cela Folie n'est rien inférieure à Amour, et qu'Amour ne serait rien sans elle: et ne peut être, et régner sans son aide" (330). Ou encore: "Jamais Amour ne fut sans la fille de Jeunesse, et ne peut être autrement" (337). Et enfin: "Amour donc ne fut jamais sans la compagnie de Folie: et ne le saurait jamais être" (348).

En effet, Mercure récuse les charges d'Apollon et la peine de séparation absolue réclamée auprès de l'assemblée divine. Folie sépare bien de lui-même le sujet possédé par l'amour-passion, mais, paradoxalement, cette rupture intérieure coïncide avec l'oubli de soi, une sorte de non-reconnaissance de soi qui rendrait possible l'union totale avec l'autre. Ce n'est qu'en s'écartant d'eux-mêmes et en changeant de forme que les amants réalisent l'unité affective intégrale. Cette disposition entraîne une alternance menaçante entre la guerre et la paix intérieures, surtout lorsque le désir d'une fusion physique et spirituelle avec l'aimé(e) rencontre des obstacles.

Or, le rôle privilégié de Folie consiste en ceci qu'elle reste toujours ouverte à l'autre, elle ne dissimule ni ne masque rien: Folie cherche moins à avoir raison d'Amour qu'à établir entre lui et elle la parfaite parité dans le dépassement du différend. L'intime déchirure vient de ce que l'être passionné doit absolument renoncer à la maîtrise, à la connaissance de soi, au bon sens et à la logique, ainsi qu'à la domination de l'objet aimé par la raison ou par le droit. Mercure déclare:

"Que te semble de Folie, Jupiter? Est-elle telle, qu'il la faille ensevelir sous le mont Gibel, ou exposer au lieu de Prométhée, sur le mont de Caucase? Est-il raisonnable la priver de toutes bonnes compagnies, où Amour sachant qu'elle sera, pour la fâcher y viendra, et conviendra [-t-il] que Folie, qui n'est rien moins qu'Amour, lui quitte sa place? S'il ne veut être avec Folie, qu'il se garde de s'y trouver. Mais que cette peine, de ne s'assembler point, tombe sur elle, ce n'est raison." (337) 
Décrivant l'issue positive de cette crise affective, Mercure affirme que le vrai amour est "grand et véhément, et plus fort que toute raison" (347). Le thème du changement de forme s'enracine dans la réciprocité symbolique entre l'amour-passion et la structure intime de l'art. La vertu cathartique de la folie serait liée à une libération de la répression rationnelle: la folie donne à rire en se communiquant à tous au-delà de la séparation. Là où le logos (représenté par Apollon dans le Débat) cherche à réprimer et à subordonner les forces de l'irrationnel selon des lois de hiérarchie et de vraisemblance, la fille de Jeunesse symbolise un retour de l'autre scène, celle du rêve et de l'imaginaire, c'est-à-dire des pulsions libidinales, dans une libre affirmation des ressemblances qui délivre le lecteur ou le spectateur des contraintes de la raison. Souvenons-nous que l'Argument du Débat précisait: "Amour se voyant poussé, entre en colère: Folie soutient lui appartenir de passer devant" (287). Que Folie dépasse Amour au seuil de la fête symbolise le mouvement par où le rêve et l'imaginaire, et par extension le milieu de la ressemblance, viennent au premier plan dans le langage littéraire, tandis que, sur le plan de l'histoire, les femmes revendiquent le droit de "mettre [leurs] conceptions par écrit" et de "passer ou égaler les hommes" en science et vertu..$^{5}$

La description labéenne de la pantomime, artifice halluciné qui se laisse entendre au-delà de la voix, indique très clairement le rapport entre l'étant et la figure. Le mime évoque les choses et les actions, il donne à voir le tout, par le moyen des "pieds et mains parlants" (335). Le mime ne montre ni le réel ni une "action effective" (Mallarmé), il y fait allusion en substituant à l'étant l'expressivité du visage et des gestes. Voici l'avis de Mercure sur les acteurs en général: "Et comment se peuvent exempter d'être nommés fols, ceux qui représentent [les anciennes fables], ayant pris, et prenant tant de peines à se faire sembler autres qu'ils ne sont?" (335). Il y a une relation décisive entre cette question de Mercure posée pour l'effet de son plaidoyer, et le thème des amants et des écrivains littéralement métamorphosés en leur autre symétrique par le désir, tout comme l'emploi de la métaphore ou de la métonymie transforme le sens et la portée des mots. ${ }^{6}$ A ce sujet, rappelons le passage relatif à la Vénus de Praxitèle que désira le jeune Cnidien, dont la sympathie pour cette "froide et morte pierre" s'enflammait, selon Mercure, grâce à la folie "logée en son esprit" (338). L'extrait qui suit celui-ci met en rapport également le délire, l’imaginaire et la ressemblance: le messager des dieux affirme que l'ardeur de Narcisse pour son propre reflet ne lui vient pas de l'oeil mais de la "folle imagination du beau pourtrait" (338). Encore une fois, la folie nomme le milieu de la figuration où se déploie le double du visage, ici l'image de la face dans l'eau, un tel 
"pourtrait" produisant un tout indissoluble au moment même où dedans et dehors apparaissent à la source dans leur différence la plus précaire.

Après le passage où Mercure démontre les effets de l'amour fou chez les hommes, le messager des dieux explique que plus les femmes ont résisté à la passion, "plus s'en trouvent prises" (342), comme si la stratégie du refus était le moyen le plus sûr d'affermir l'attachement à l'être aimé. Cette thématique est liée à l'articulation du désir: les femmes aux prises avec la folie amoureuse "prennent la plume et le luth en main: écrivent et chantent leurs passions" (343), ou comme Mercure (associé aussi à l'invention de l'alphabet et du jeu de dés) dira plus loin: "Plusieurs femmes, pour plaire à leurs Poètes amis, ont changé leurs paniers et coutures, en plumes et livres" (346-47). Labé marque ainsi le rapport étroit entre la passion et l'imaginaire, dans la mesure où Folie prépare les coeurs et pour les flèches d'Amour, et pour la plume. Dans les deux cas, on passe par le stade de la dénégation où le désir de rester intégralement soi-même, de perpétuer son identité à soi, paradoxalement dispose l'être humain aimant à la volonté de s'abandonner et de se livrer corps et âme à l'autre. Jusqu'à un certain point, la possibilité d'un grand amour partagé apparaît comme une menace à l'intégrité de soi, puis par un mécanisme que Labé ramène à la folie, on saute au pôle opposé en passant outre même à la peur. "Elles ferment la porte à [la] raison. Tout ce qu'elles craignaient, ne le [re]doutent plus" (342). Tel passage d'un extrême à l'autre dénote la sortie hors-de-soi, l'oubli ou la non-connaissance de soi, qui mènent au changement de forme, à la métamorphose nécessaire à l'épanouissement de la passion.

Doit-on séparer chronologiquement le moment du refus de l'amour et celui de l'abandon? Le refus est un moment essentiel du don dans la mesure où le moi se constitue ainsi dans son identité en même temps qu'il cherche à sortir de soi, et on peut dire que le propre du texte littéraire est que la constitution d'un sujet unitaire présuppose la fusion du moi réel avec son autre radical: l'écriture. Dans l'amant comme dans l'écriture, la persona voit un double qui à la fois l'éloigne d'un moi conventionnel et la fait accéder au moi authentique capable de toutes les émotions possibles. Or, se figurer ainsi par l'image de l'autre serait un signe de folie: la volonté de se voir autre que soi, de se faire tout autre ne mène pas par elle-même au savoir ou à la connaissance, mais au contraire à l'expérience préparatoire de 1"étrange et forte passion" (392) qui, chez Labé, donne lieu à l'activité créatrice. Ce rapport de soi à l'autre figuré par le poème comme espace ou marge de la différence apparaît bien dans son sonnet des antithèses. Il est vrai que le mode de ce poème se rattache à une tradition qui passe par 
Pétrarque, Villon et Du Bellay, mais La Belle a soy en donne une illustration nouvelle en l'inscrivant dans le contexte de la folie amoureuse telle que sa propre expérience l'a déterminée. Ce poème constitue l'un des jalons principaux de la séquence des sonnets car il marque le point extrême auquel aboutit "la dialectique par définition tumultueuse de l'extase et du déchirement alternant au sein de 'l'amour-passion"' (Berriot, 92):

Je vis, je meurs: je me brûle et me noie.

J'ai chaud estrême en endurant froidure:

La vie m'est et trop molle et trop dure.

J'ai grands ennuis entremêlés de joie:

Tout à un coup je ris et je larmoie,

Et en plaisir maint grief tourment j'endure:

Mon bien s'en va, et à jamais il dure;

Tout en un coup je sèche et je verdoie. (VIII, 376)

C'est à partir du moment où l'écrivain passe dans le domaine du paradoxe qu'elle sera capable de ressentir le tout de l'affectivité en même temps: la "partie" qu'est un moment privilégié d'amour ouvre sur la totalité de la jouissance et de la douleur, du bien et du mal, de même que l'écrit fait revenir la valeur de la présence en l'exposant au bord de la disparition et de l'oubli. La persona de la femme amoureuse ressent dans ses fibres le risque pris de son désir éperdu qui est à la fois une extrême concentration de sa volonté et une passion subie en pure perte.

Le Sonnet XVII offre un exemple saisissant de l'amour fou tel que Labé l'envisage, car il dévoile l'image de la sortic hors-de-soi, caractéristique de la folie, par une tournure polysémique qui en inscrit l'essence dans la syntaxe même du dernier vers. La persona fuit ici "la présence obsédante de l'aimé" (Berriot, 385), en tâchant de se faire un nouvel objet et de se distraire des "pensers amoureux" (XVII, v. 9). Karine Berriot interprète le dernier tercet comme un dénouement à double sens, où l'amante ne pourrait se délivrer de sa hantise qu'en allant vivre en celui qui occupe sa pensée. Cette lecture rejoint notre propos, car il met en relief le moment où le sujet, ayant refusé le don de soi, reconnaît que la seule issue possible à la passion obsédante serait l'abandon total au désir. Mais le dernier vers laisse en suspens le sujet du verbe être au subjonctif, comme si le langage poétique incorporait par là le phénomène du vivre-l'un-en-l'autre qui constitue l'issue thématique du sonnet: 
Que si je veux de toi être délivre,

Il me convient hors de moi-même vivre,

Ou fais encor que loin sois en séjour. (XVII, 385)

Eloignement et proximité des amants reviennent ici au même, comme le rapprochement de deux étants distincts peut produire une figure où la différence s'affirme en tant que "milieu de la relation infinie" (Heidegger) dans la langue (c'est l'entre-deux ou le neutre). ${ }^{7}$

Un langage fou (on pense à Blanchot parlant de Hölerlin) traverse de part en part les Oeuvres de Labé, mais il y a encore un texte où la sortie hors-de-soi s'opère explicitement en rapport avec l'érotisme et la spiritualité. Il s'agit du célèbre "sonnet des baisers" qui pose la réciprocité (et donc la perte de l'identité intrinsèque) des deux amants par les baisers sensuels, comme une folie qui donne accès à la double vie. Labé réinterprète un motif néo-platonicien en soutenant l'exigence de la réciprocité des corps, car la jouissance mutuelle ici s'intègre à l'échange proprement spirituel. François Rigolot suggère que le jeu réciproque des deux langues par rapport à la double vie spirituelle répète la relation structurale entre l'inscription et le sens des paroles. ${ }^{8}$ Labé pense l'unité du corps et de l'esprit en termes d'une folie créatrice qui rend possible le devenir-autre du sujet (le "JE est un autre" de Rimbaud):

Lors double vie à chacun en suivra.

Chacun en soi et son ami vivra.

Permets m'Amour penser quelque folie:

Toujours suis mal, vivant discrètement,

Et ne me puis donner contentement,

Si hors de moi ne fais quelque saillie. (XVIII, 386)

Ce que chante Labé dans ce sonnet à la fois érotique et méditatif, c'est l'un-en-deux du rapport amoureux, la réciprocité totale du désir éperdu qui suspend l'identité individuelle des amants dans l'échange sensuel, unique source de jouissance entendue comme l'allégresse de tout attachement affectif extrême. En concevant le dépassement de la séparation douloureuse, la persona ici s'apprête à se donner du "contentement": elle trouvera le bonheur en vivant intensément en soi ET pour l'autre, ce que le poème met en scène par ses figures et polysémies qui jaillissent hors de l'identité simple d'un contenu sémantique.

Pour nous, l'intérêt multiple de l'oeuvre labéenne consiste en sa figuration incomparable de l'amour fou, laquelle inscrit à la fois une pensée féministe féconde et une réflexion sur le langage poétique au-delà de la raison. On 
a vu que dans l'Epître dédicatoire, Labé juxtapose, à dessein, les sphères décisives de l'affirmation féminine et de la reconstitution imaginative de l'expérience et du savoir. La Lyonnaise a consciemment pensé le rapport des termes de ce qui se nomme aujourd'hui écriture au féminin. ${ }^{9}$ En tant que la persona des Oeuvres s'associe à la déesse Folie aussi bien qu'à la thématique du délire amoureux, les textes de Labé nous ramènent au centre paradoxal où la présence s'affirme à travers l'oubli, la jouissance à travers la douleur, la paix à travers le plus explosif des conflits psychiques. Ainsi La Belle a soy nous a-t-elle légué un recueil élégant et profond témoignant de l'unité originaire entre l'expérience passionnée de la femme écrivain et l'essence discrètement ouverte de l'écriture poétique.

\section{Wichita State University}

1 Citons ici les vers bien connus de la première élégie de Labé, où la métamorphose de la persona est comparée à celle de la Reine Sémiramis en proie à une passion incestueuse: "Ainsi Amour de toi t'a étrangée / Qu'on te dirait en une autre changée" (356). Les numéros de page entre parenthèses renvoient à Karine Berriot, Louise Labé: La Belle Rebelle et le François nouveau suivi des Oeuvres complètes (Seuil, 1985). Une première version de la présente analyse a été lue au congrès du Conseil International d'Etudes Francophones à Montréal, le 16 avril 1988.

2 C'est au cours de son plaidoyer en faveur de Folie dans le Débat de Folie et d'Amour (337) que Mercure prononce cette formule très suggestive, qui ramène l'origine de l'Amour (à la fois le sentiment et son incarnation mythologique) à la Folie. La pensée voilée du syntagme met en rapport l'aube de l'amour (dont l'un des noms est Vénus, mère de Cupidon, beauté née de la mer comme l'aurore) et l'éternel retour ("la belle première naissance," répétée au XVIe siècle français, étant aussi Renaissance).

3 Cette expression que recommande Nicole Brossard dans ses conférences se trouve par exemple dans la notice biographique de Double Impression (L'Hexagone, 1984), p.139.

4 Voir l'Epître dédicatoire, p.283. Communiquée souvent de manière implicite, la conception labéenne de la différence (temporelle, sexuelle, poétique, etc.) s'éclaire, selon nous, à la lecture des textes suivants: Martin Heidegger, "Identité et différence," dans Questions I (Gallimard, 1968), pp.253-308; Gilles Deleuze, Différence et répétition (Presses Universitaires de France, 1968); Jacques Derrida, "La différance," in Marges de la philosophie (Minuit, 1972), pp.3-29; René Girard, Des choses cachées depuis la fondation du monde (Grasset, 1978); Michel Deguy, Choses de la poésie et affaire culturelle (Hachette, 1986). Quant au statut du discours de la folie, il convient de renvoyer à Michel Foucault, Folie et déraison: Histoire de la folie d̀ l'áge classique (Plon, 1961), et à Maurice Blanchot, Le pas au-delà (Gallimard, 1973). La présente analyse tente de comprendre les motifs ci-dessus mentionnés en rapport avec les perspectives féministes exposées par Adrienne Rich dans On Lies, Secrets, and Silence (Norton, 1979).

5 Voir l'Epitre dédicatoire, pp.281-82.

6 Pour une analyse percutante du rapport entre les figures de comparaison et l'opposition Amour/Folie chez Labé, voir R.D. Cottrell, "The Problematics of Opposition in Louise Labe's Débat de Folie et d'Amour," French Forum, 12 (1987), 27-42.

7 Cr. Michel Deguy, Figurations (Gallimard, 1969), p.147. 
8 Voir François Rigolot, "Signature et signification: Les baisers de Louise Labé," Romanic Review, 75 (1984), 10-24.

9 Cf. la note 3 sup. L'essai remarquable de Karine Berriot montre que Labé tient également un discours politique dans la mesure où Apollon et Mercure, Amour et Folie représentent respectivement le patriarcat et le monde des femmes, l'aristocratie et la nouvelle bourgeoisie marchande: une femme bourgeoise revendique son droit à l'amour noble et annonce un courant fondamental de l'imagination créatrice moderne. Voir Berriot, pp.66-99 et passim. 